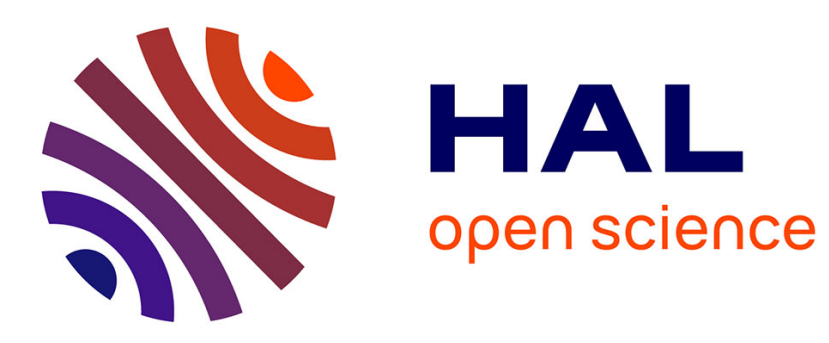

\title{
On finite-time stability analysis of homogeneous Persidskii systems using LMIs
}

Konstantin Zimenko, Denis Efimov, Andrey Polyakov, Artem Kremlev

\section{To cite this version:}

Konstantin Zimenko, Denis Efimov, Andrey Polyakov, Artem Kremlev. On finite-time stability analysis of homogeneous Persidskii systems using LMIs. Proc. 60th IEEE Conference on Decision and Control (CDC), Dec 2021, Austin, United States. hal-03439205

\section{HAL Id: hal-03439205 https://inria.hal.science/hal-03439205}

Submitted on 22 Nov 2021

HAL is a multi-disciplinary open access archive for the deposit and dissemination of scientific research documents, whether they are published or not. The documents may come from teaching and research institutions in France or abroad, or from public or private research centers.
L'archive ouverte pluridisciplinaire HAL, est destinée au dépôt et à la diffusion de documents scientifiques de niveau recherche, publiés ou non, émanant des établissements d'enseignement et de recherche français ou étrangers, des laboratoires publics ou privés. 


\title{
On finite-time stability analysis of homogeneous Persidskii systems using LMIs*
}

\author{
Konstantin Zimenko ${ }^{\dagger}$, Denis Efimov ${ }^{\ddagger, \dagger}$, Andrey Polyakov ${ }^{\ddagger}, \dagger$ and Artem Kremlev ${ }^{\dagger}$
}

\begin{abstract}
The paper provides a sufficient condition to ensure robust finite-time stability of homogeneous generalized Persidskii systems. The proposed condition is formulated using linear matrix inequalities and it allows to obtain settling time estimates. The results are supported with numerical examples.
\end{abstract}

\section{INTRODUCTION}

Homogeneity is a kind of symmetry of the right-hand sides of dynamical systems implying also a similar property for their solutions, which is widely used in systems and control theory for stability and robustness analysis, nonlinear control and observer design, etc. (see, for example, [1][12]). In addition, homogeneous differential equations and inclusions describe a (local or approximate) behaviour of a wide class of dynamical systems, which includes some physical phenomena (e.g., flow of heat, motion of viscous fluids [3]), nonholonomic systems [13], mechanical models with frictions [14], etc. The use of the homogeneity property in regulator and observer design is supported by a number of useful features, such as behaviour scalability (local (stability) property can be transformed to the whole state space), nonasymptotic convergence rates (finite-time or fixed-time stability) and robustness (e.g., Input-to-State Stability (ISS) and delay robustness) [2], [6], [7].

Stability analysis of nonlinear systems is a basic problem in systems and control theory whose solution is based on the use of Lyapunov function method, then it is hard to solve this problem constructively in a general case since there is no common approach to select a Lyapunov function [19]. In the case of homogeneous systems, in [10], it was shown that such systems are equivalent to standard homogeneous ones (namely, the systems $\dot{x}=f(x)$, where $f(\lambda x)=\lambda f(x)$ for all $\lambda>0$ ) via appropriate change of coordinates (diffeomorphism). Based on this equivalence it was shown that for a stable homogeneous system there exists an implicitly defined quadratic-like homogeneous Lyapunov function. The choice of such a Lyapunov function may sufficiently simplify stability analysis for many practical cases (see [10]), and in particular the Implicit Lyapunov Function (ILF) method was

*This work was supported by the Russian Science Foundation under grant 21-71-10032.

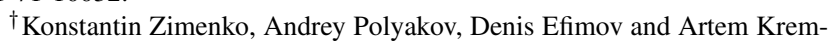
lev are with Faculty of Control Systems and Robotics, ITMO University, 49 Kronverkskiy av., 197101 Saint Petersburg, Russia.

${ }^{\ddagger}$ Denis Efimov and Andrey Polyakov are with Inria, Univ. Lille, CNRS, UMR 9189 - CRIStAL, F-59000 Lille, France.

e-mail: konstantin.zimenkoditmo.ru,

denis.efimoveinria.fr,

andrey.polyakoveinria.fr,kremlev_artememail.ru applied to design of finite-/fixed-time stabilizing controls of linear systems [4], [5].

Pursuing the aim to enlarge the class of the systems to which the ILF method can be applied, the present paper deals with the class of generalized Persidskii systems that are homogeneous, and have a linear part and different nonlinearities. Persidskii systems were studied in the context of neural networks [15], [16], electric circuits [24], robust stability analysis [17], gradient systems convergence analysis [18], etc. In [17] stability criteria was presented in the form of Linear Matrix Inequalities (LMIs). However, in general case, these LMIs are infeasible for homogeneous Persidskii systems due to homogeneity imposes some restrictions on system matrices (e.g., linear part of the system is presented or can be reduced to the form $A_{0} x, x \in \mathbb{R}^{n}$ with a nilpotent matrix $A_{0} \in \mathbb{R}^{n \times n}$ ), and the approach of [17] does not take into account a possible homogeneity of the system, then the used Lyapunov function cannot be homogeneous. In some cases this problem can be handled with the use of homogeneous approximations as in [25]. However, this approach does not allow to obtain settling time estimations and does not provide quantitative robustness analysis. The main goal of this paper is to propose a new constructive modification of the ILF approach by formulating LMIs to check finite-time stability for homogeneous Persidskii systems with negative degree; and provide both quantitative and qualitative robustness analysis.

More precisely, in this work for homogeneous Persidskii systems an implicit homogeneous Lyapunov function is proposed, where a properly dilated sum of quadratic-like part and linear combination of the integrals of the nonlinearities is used. This form of Lyapunov function allows to obtain simple sufficient stability conditions in terms of LMIs. Note, that with the use of the local homogeneity concept the presented result can be developed for nonhomogeneous systems to provide local finite-time stability analysis.

The outline of this paper is as follows. Notation used in the paper is given in Section II. Preliminary results are introduced in Section III. The problem statement is given in Section IV. Section V presents the main result on finite-time stability conditions for homogeneous Persidskii systems. Numerical example is given in Section VI. Finally, concluding remarks are discussed in Section VII. 


\section{NOTATION}

Through the paper the following notation will be used:

- $\mathbb{R}_{+}=\{x \in \mathbb{R}: x>0\}$, where $\mathbb{R}$ is the field of real numbers;

- $\mathbb{R}^{n}$ denotes the $n$ dimensional Euclidean space with vector norm $\|\cdot\|$;

- The symbol $\overline{1, m}$ is used to denote a sequence of integers $1, \ldots, m$;

- The order relation $P>0(<0 ; \geq 0 ; \leq 0)$ for $P \in$ $\mathbb{R}^{n \times n}$ means that $P$ is symmetric and positive (negative) definite (semidefinite);

- $\lambda_{\max }(P)$ and $\lambda_{\min }(P)$ denote maximum and minimum eigenvalues of a symmetric matrix $P \in \mathbb{R}^{n \times n}$;

- The set of nonnegative diagonal matrices with dimension $n \times n$ is defined by $\mathbb{D}_{+}^{n}$;

- $\operatorname{diag}\left\{\lambda_{i}\right\}_{i=1}^{n}$ is a diagonal matrix with elements $\lambda_{i}, i=$ $\overline{1, n}$

- $\mathrm{O}_{m \times n}$ denotes zero matrix with dimensions $m \times n$;

- $\mathcal{L}_{\infty}$ is the set of essentially bounded measurable functions $\mathbb{R} \rightarrow \mathbb{R}^{m}$ with the norm $\|\cdot\|_{[0,+\infty)}=$ $\sup _{t \in[0,+\infty)}\|\cdot(t)\|$.

\section{PRELIMINARIES}

Let us consider the system

$$
\dot{x}=f(x), \quad x(0)=x_{0},
$$

where $x \in \mathbb{R}^{n}$ is the state vector, $f: \mathbb{R}^{n} \rightarrow \mathbb{R}^{n}$ is a vector field, $f(0)=0$. If the vector field $f$ is discontinuous with respect to $x$, then the solutions of (1) fall into the area of differential inclusions and need to be understood in the sense of Filippov [20]. Assume that the system (1) has unique solutions defined in forward time (at least locally).

\section{A. Finite-time stability}

Definition 1 [21], [14] The origin of (1) is said to be globally finite-time stable if it is globally asymptotically stable and any solution $x\left(t, x_{0}\right)$ of the system (1) reaches the equilibrium point at some finite time moment, i.e. $x\left(t, x_{0}\right)=$ $0 \forall t \geq T\left(x_{0}\right)$ and $x\left(t, x_{0}\right) \neq 0 \forall t \in\left[0, T\left(x_{0}\right)\right), x_{0} \neq 0$, where $T: \mathbb{R}^{n} \rightarrow \mathbb{R}_{+} \cup\{0\}, T(0)=0$ is the settling-time function.

The following theorem presents the ILF method [22], [23] for finite-time stability analysis.

Theorem 1 [4] Suppose that there exists a continuous function

$$
\begin{aligned}
& Q: \mathbb{R}_{+} \times \mathbb{R}^{n} \rightarrow \mathbb{R} \\
& (V, x) \mapsto Q(V, x)
\end{aligned}
$$

such that

C1) $Q(V, x)$ is continuously differentiable $\forall x \in \mathbb{R}^{n} \backslash\{0\}$ and $\forall V \in \mathbb{R}_{+}$;

C2) for any $x \in \mathbb{R}^{n} \backslash\{0\}$ there exist $V^{-} \in \mathbb{R}_{+}$and $V^{+} \in$ $\mathbb{R}_{+}$:

$$
Q\left(V^{-}, x\right)<0<Q\left(V^{+}, x\right)
$$

C3) for $\Omega=\left\{(V, x) \in \mathbb{R}^{n+1}: Q(V, x)=0\right\}$

$$
\lim _{\substack{x \rightarrow 0 \\(V, x) \in \Omega}} V=0, \lim _{\substack{V \rightarrow 0^{+} \\(V, x) \in \Omega}}\|x\|=0, \lim _{\substack{\|x\| \rightarrow \infty \\(V, x) \in \Omega}} V=+\infty ;
$$

C4) the inequality

$$
-\infty<\frac{\partial Q(V, x)}{\partial V}<0
$$

holds $\forall V \in \mathbb{R}_{+}$and $\forall x \in \mathbb{R}^{n} \backslash\{0\}$;

C5) the inequality

$$
\frac{\partial Q(V, x)}{\partial x} f(x) \leq \alpha V^{1+\mu} \frac{\partial Q(V, x)}{\partial V}
$$

holds $\forall(V, x) \in \Omega$, where $\mu<0$ and $\alpha>0$ are some constants.

Then the origin of the system (1) is globally finite-time stable and

$$
T\left(x_{0}\right) \leq-\frac{V_{0}^{-\mu}}{\alpha \mu},
$$

where $Q\left(V_{0}, x_{0}\right)=0$.

\section{B. Weighted homogeneity}

The homogeneity is a symmetry property of an object with respect to a group of transformations (dilation operation). This property provides many advantages to analysis (including finite-time stability and robustness properties) and design of nonlinear control system.

For $r_{i}, \lambda \in \mathbb{R}_{+}, i=\overline{1, n}$ define the dilation matrix $D_{r}(\lambda)=\operatorname{diag}\left\{\lambda^{r_{i}}\right\}_{i=1}^{n}$ and the vector of weights $r=$ $\left[\begin{array}{lll}r_{1}, & \ldots, r_{n}\end{array}\right]^{T} \in \mathbb{R}_{+}^{n}, r_{\text {max }}=\max _{1 \leq j \leq n} r_{j}, r_{\text {min }}=$ $\min _{1 \leq j \leq n} r_{j}$.

Definition 2 [1] A function $g: \mathbb{R}^{n} \rightarrow \mathbb{R}$ (vector field $f: \mathbb{R}^{n} \rightarrow \mathbb{R}^{n}$ ) is said to be r-homogeneous of degree $\mu$ if

$$
\begin{gathered}
g\left(D_{r}(\lambda) x\right)=\lambda^{\mu} g(x) \\
\left(f\left(D_{r}(\lambda) x\right)=\lambda^{\mu} D_{r}(\lambda) f(x)\right)
\end{gathered}
$$

for given $r$, all $\lambda \in \mathbb{R}_{+}$and all $x \in \mathbb{R}^{n}$.

The following theorem provides the relation of homogeneity property and finite-time stability:

Theorem 2 [9] Let $f: \mathbb{R}^{n} \rightarrow \mathbb{R}^{n}$ be a r-homogeneous vector field with degree $\mu<0$. If the origin of the system

$$
\dot{x}=f(x)
$$

is locally asymptotically stable, then it is globally finite-time stable and there exists a continuously differentiable Lyapunov function $V$ which is $r$-homogeneous of degree $v>-\mu$.

Under homogeneity conditions, asymptotic stability of a nonlinear system with zero disturbance implies a certain robustness in ISS or integral ISS sense. Consider the system

$$
\dot{x}(t)=f(x(t), d(t)), \quad t \geq 0,
$$

where $x(t) \in \mathbb{R}^{n}$ is the state, $d(t) \in \mathbb{R}^{m}$ is the external input, $d \in \mathcal{L}_{\infty}$, and $f: \mathbb{R}^{n} \times \mathbb{R}^{m} \rightarrow \mathbb{R}^{n}$ is a locally Lipschitz (or Hölder) continuous function, $f(0,0)=0$.

Lemma 1 [2] Let the vector field $\tilde{f}(x, d)=$ $\left[f(x, d)^{T}, O_{1 \times m}\right]^{T}$ be homogeneous with the weights $r=$ $\left[\begin{array}{lll}r_{1}, & \ldots, & r_{n}\end{array}\right]^{T}>0, \quad \tilde{r}=\left[\begin{array}{lll}\tilde{r}_{1}, & \ldots, & \tilde{r}_{m}\end{array}\right]^{T} \geq 0$ with a degree $\mu \geq-r_{\text {min }}$, i.e., $f\left(D_{r}(\lambda) x, D_{\tilde{r}}(\lambda) d\right)=$ $\lambda^{\mu} D_{r}(\lambda) f(x, d)$ for all $x \in \mathbb{R}^{n}, d \in \mathbb{R}^{m}$ and all $\lambda>0$. Assume that the system (4) is globally asymptotically stable 
for $d=0$, then the system (4) is

ISS if $\tilde{r}_{\text {min }}>0$, where $\tilde{r}_{\text {min }}=\min _{1 \leq j \leq m} \tilde{r}_{j}$; integral ISS if $\tilde{r}_{\min }=0$ and $\mu \leq 0$.

The $r$-homogeneity presented in Definition 2 is introduced for some $r \in \mathbb{R}_{+}^{n}$ and all $\lambda \in \mathbb{R}_{+}$. Restricting the set of admissible values for $\lambda$ the local homogeneity concept has been introduced in [1], [2], [8].

\section{PROBLEM STATEMENT}

Consider the following class of systems:

$$
\dot{x}(t)=A_{0} x(t)+\sum_{j=1}^{M} A_{j} f^{j}(x(t))+d(t, x(t)), \quad t \geq 0,
$$

where $x(t)=\left[x_{1}(t) \ldots x_{n}(t)\right]^{T} \in \mathbb{R}^{n}$ is the state vector, $x(0) \in \mathbb{R}^{n}, f^{j}(x)=\left[f_{1}^{j}\left(x_{1}\right) \ldots f_{n}^{j}\left(x_{n}\right)\right]^{T}, j=\overline{1, M}$ are the functions ensuring existence of solutions of the system (5) in forward time, $f^{j}(x)$ is continuous, $f^{j}(0)=0$, the matrices $A_{k} \in \mathbb{R}^{n \times n}$ for $k=\overline{0, M}$, and the function $d: \mathbb{R} \times \mathbb{R}^{n} \rightarrow \mathbb{R}^{n}$ describes exogenous disturbances and uncertainties.

The diagonal structure of the nonlinearities (i.e., that $f_{i}^{j}(x)=f_{i}^{j}\left(x_{i}\right)$ for $j=\overline{1, M}$ and $i=\overline{1, n}$ ), places (5) to a class of Persidskii systems. It is assumed that the system (5) satisfies the following assumptions:

\section{Assumption 1}

For any $i=\overline{1, n}, j=\overline{1, M}$ :

$$
s f_{i}^{j}(s)>0 \quad \forall s \in \mathbb{R} \backslash\{0\} .
$$

Assumption 2 The system (5) with $d=0$ is $r$ homogeneous of degree $\mu<0$, i.e.

$$
\begin{gathered}
D_{r}(\lambda)\left(A_{0} x+\sum_{j=1}^{M} A_{j} f^{j}(x)\right) \\
=\lambda^{-\mu}\left(A_{0} D_{r}(\lambda) x+\sum_{j=1}^{M} A_{j} f^{j}\left(D_{r}(\lambda) x\right)\right) ;
\end{gathered}
$$

and there exists $\rho \in\{1, \ldots, M\}$ such that $f^{j}(x)$ are homogeneous of degree $\mu$ for $j=\overline{1, \rho}$.

In Assumption 1 it is stated that all nonlinearities belong to a sector and may take zero values at zero only. Assumption 2 restricts (5) to the class of disturbed homogeneous Persidskii systems. The presence of homogeneous functions $f^{j}(x)$, $j=\overline{1, \rho}$ can be obtained after a proper re-indexing and decomposition of a nonlinear system dynamics, and the principal restriction of Assumption 2 is (7).

Remark 1 According to Theorem 2, to establish the finitetime stability property of (5) with $d=0$ it is enough to show asymptotic stability of the system. In this case, at a first glance, to solve the problem we can use sufficient LMIbased conditions as in [17] to check asymptotic stability, and then the system is finite-time stable due to homogeneity property. According to [17] one of the main requirements for stability checking is that there should exist matrices $P=$ $P^{T} \in \mathbb{R}^{n}, P \geq 0$ and $\Lambda^{j} \in \mathbb{D}_{+}^{n}$ for $j=\overline{1, M}$ to serve as solutions of the Lyapunov equation for the matrices $A_{0}$ and $A_{j}$ for $j=\overline{1, M}$, respectively. However, this approach has the following sticking point: for nonzero linear part of the system (5) the homogeneity property imposes restrictions on the system matrices ( $A_{0}$ is nilpotent or $A_{j}$ is not diagonally stable for some $j \in\{1, \ldots, M\}$ ), that is difficult to fit into the requirements of [17]. Thus, such an approach can be used only for $A_{0}=0$, that significantly reduces the class of systems under consideration.

This paper is aimed to derive constructive (given in the LMI form) conditions of robust finite-time stability of the homogeneous system (5) with a settling-time estimate.

\section{MAIN RESULT}

After proper re-indexing and decomposition of a nonlinear system dynamics the sector condition (6) and homogeneity property imply that:

- for any $i=\overline{1, n}, j=\overline{1, \zeta}$ the nonlinearities $f_{i}^{j}$ have unbounded integrals, i.e.,

$$
\lim _{s \rightarrow \pm \infty} \int_{0}^{s} f_{i}^{j}(\sigma) d \sigma=+\infty
$$

where $\zeta \geq \rho$;

- due to negative homogeneity degree $\mu$ for any $i=\overline{1, n}$, $j=\overline{1, \rho}$ :

$$
s f_{i}^{j}(s)>\psi_{i}^{j} s^{2} \quad \forall s \in\left[\underline{s}_{i}, \bar{s}_{i}\right] \backslash\{0\},
$$

where $\psi_{i}^{j} \in \mathbb{R}_{+}$and $-\infty<\underline{s}_{i}<0<\bar{s}_{i}<+\infty$.

Define $\Psi^{j}=\operatorname{diag}\left\{\psi_{i}^{j}\right\}_{i=1}^{n} \in \mathbb{D}_{+}^{n}, j=\overline{1, \rho}$.

Introduce the function in the form

$$
\begin{aligned}
Q(V, x)= & x^{T} D_{r}\left(V^{-1}\right) P D_{r}\left(V^{-1}\right) x+ \\
& 2 \sum_{j=1}^{M} \sum_{i=1}^{n} \Lambda_{i}^{j} \int_{0}^{V^{-r_{i}} x_{i}} f_{i}^{j}(s) d s-\chi,
\end{aligned}
$$

where $\Lambda^{j}=\operatorname{diag}\left\{\Lambda_{i}^{j}\right\}_{i=1}^{n} \in \mathbb{D}_{+}^{n}, P \in \mathbb{R}^{n \times n}$ is a symmetric positive semi-definite matrix, and $\chi \in \mathbb{R}_{+}$. All these quantities will be selected below as a solution of LMI. For $\Lambda^{j}=0$, $j=\overline{1, M}$ the same equation for ILF was considered in [4], [4] for the chain of integrators.

Denote $H=\operatorname{diag}\left\{r_{i}\right\}_{i=1}^{n}$.

The main result of this paper is as follows:

Theorem 3 Let Assumptions 1, 2 be satisfied. Let $\alpha \in \mathbb{R}_{+}$, $\iota \in \mathbb{R}$ be chosen such that the LMI system

$$
\begin{aligned}
& P \geq 0 ; \quad \Gamma>0 ; \quad P+2 \sum_{j=1}^{\zeta} \Lambda^{j}>0 ; \\
& H P+P H \geq 0 ; \quad H \sum_{j=1}^{M} \Lambda^{j}+\iota(H P+P H)>0 ; \\
& Q_{1}+\left[\begin{array}{cc}
Q_{3} & \mathrm{O}_{(M+1) n \times n} \\
\mathrm{O}_{n \times(M+1) n} & \mathrm{O}_{n \times n}
\end{array}\right]+ \\
& \alpha\left[\begin{array}{cc}
Q_{2} & \mathrm{O}_{(M+1) n \times n} \\
\mathrm{O}_{n \times(M+1) n} & \mathrm{O}_{n \times n}
\end{array}\right] \leq 0,
\end{aligned}
$$

is feasible for some $P=P^{T}, \quad \Gamma=\Gamma^{T} \in \mathbb{R}^{n \times n}$; $\Xi^{k}, \Lambda^{j}, \Upsilon_{\eta, l} \in \mathbb{D}_{+}^{n}, k=\overline{0, M}, j=\overline{1, M}, \eta=\overline{0, M-1}$, $l=\overline{\eta+1, M}$, where for $X=P+\sum_{j=1}^{\rho} \Lambda^{j} \Psi^{j}$ and $\tilde{A}_{0}=A_{0}+\sum_{j=1}^{\rho} A_{j} \Psi^{j}$

$$
Q_{1}=\left[\begin{array}{ccccc}
X \tilde{A}_{0}+\tilde{A}_{0}^{T} X & X A_{1}+\tilde{A}_{0}^{T} \Lambda^{1} & \cdots & X A_{M}+\tilde{A}_{0}^{T} \Lambda^{M} & X \\
\Lambda^{1} \tilde{A}_{0}+A_{1}^{T} X & \Lambda^{1} A_{1}+A_{1}^{T} \Lambda^{1} & \cdots & \Lambda^{1} A_{M}+A_{1}^{T} \Lambda^{M} & \Lambda^{1} \\
\vdots & \vdots & \ddots & \vdots & \vdots \\
\Lambda^{M} \tilde{A}_{0}+A_{M}^{T} X & \Lambda^{M} A_{1}+A_{M}^{T} \Lambda^{1} & \cdots & \Lambda^{M} A_{M}+A_{M}^{T} \Lambda^{M} & \Lambda^{M} \\
X & \Lambda^{1} & \cdots & \Lambda^{M} & -\Gamma
\end{array}\right] ;
$$




$$
\begin{gathered}
Q_{2}=\left[\begin{array}{cccc}
X H+H X & \Lambda^{1} H & \cdots & \Lambda^{M} H \\
\Lambda^{1} H & & \\
\vdots & \bigcup_{M n \times M n} \\
\Lambda^{M} H &
\end{array}\right] \\
Q_{3}=\left[\begin{array}{cccc}
\Xi^{0} & \Upsilon_{0,1} & \cdots & \Upsilon_{0, M} \\
\Upsilon_{0,1}^{T} & \Xi^{1} & \cdots & \Upsilon_{1, M} \\
\vdots & \vdots & \ddots & \vdots \\
\Upsilon_{0, M}^{T} & \Upsilon_{1, M}^{T} & \cdots & \Xi^{M}
\end{array}\right]
\end{gathered}
$$

and let

$$
\begin{gathered}
d^{T} D_{r}\left(V^{-1}\right) \Gamma D_{r}\left(V^{-1}\right) d \leq V^{2 \mu} y^{T} Q_{3} y \\
\text { for } y=\left[\begin{array}{c}
D_{r}\left(V^{-1}\right) x \\
f^{1}\left(D_{r}\left(V^{-1}\right) x\right)-\Psi^{1} D_{r}\left(V^{-1}\right) x \\
\vdots \\
f^{\rho}\left(D_{r}\left(V^{-1}\right) x\right)-\Psi^{\rho} D_{r}\left(V^{-1}\right) x \\
f^{\rho+1}\left(D_{r}\left(V^{-1}\right) x\right) \\
\vdots \\
f^{M}\left(D_{r}\left(V^{-1}\right) x\right)
\end{array}\right] \text { and } V \in \mathbb{R}_{+}: \\
Q(V, x)=0 \text { with } \chi<\min _{i \in \overline{1, n}}\left\{\underline{s}_{i}^{2}, \bar{s}_{i}^{2}\right\} \lambda_{\min }(X) . \\
\text { Then the origin is globally finite-time stable and }
\end{gathered}
$$

$$
T\left(x_{0}\right) \leq-\frac{V_{0}^{-\mu}}{\alpha \mu},
$$

where $Q\left(V_{0}, x_{0}\right)=0$.

All proofs are skipped due to space limitations.

Remark 2 The result of Theorem 3 is based on the use of the following system representation:

$$
\begin{aligned}
\dot{x}(t)= & \left(A_{0}+\sum_{j=1}^{\rho} A_{j} \Psi^{j}\right) x(t)+ \\
& \sum_{j=1}^{\rho} A_{j}\left(f^{j}(x(t))-\Psi^{j} x(t)\right)+ \\
& \sum_{m=\rho+1}^{M} A_{m}\left(f^{m}(x(t))\right)+d(t, x(t)), \quad t \geq 0,
\end{aligned}
$$

that was applied in order to obtain more efficient LMIs (see Remark 3 below).

Stable homogeneous systems have such qualitative stability properties as ISS with respect to additive perturbations and measurement noises (see, for example [2]). In this case to establish the ISS property it is enough to analyse system stability with $d=0$.

Corollary 1 Let all conditions of Theorem 3 be satisfied for $\Gamma=0$ and

$Q_{1}=\left[\begin{array}{ccccc}X \tilde{A}_{0}+\tilde{A}_{0}^{T} X & X A_{1}+\tilde{A}_{0}^{T} \Lambda^{1} & \cdots & X A_{M}+\tilde{A}_{0}^{T} \Lambda^{M} & \mathrm{O}_{n \times n} \\ \Lambda^{1} \tilde{A}_{0}+A_{1}^{T} X & \Lambda^{1} A_{1}+A_{1}^{T} \Lambda^{1} & \cdots & \Lambda^{1} A_{M}+A_{1}^{T} \Lambda^{M} & \mathrm{O}_{n \times n} \\ \vdots & \vdots & \ddots & \vdots & \vdots \\ \Lambda^{M} \tilde{A}_{0}+A_{M}^{T} X & \Lambda^{M} A_{1}+A_{M}^{T} \Lambda^{1} & \cdots & \Lambda^{M} A_{M}+A_{M}^{T} \Lambda^{M} & \mathrm{O}_{n \times n} \\ \mathrm{O}_{n \times n} & \mathrm{O}_{n \times n} & \cdots & \mathrm{O}_{n \times n} & \mathrm{O}_{n \times n}\end{array}\right]$.

Then the system (5) is ISS for $d \in \mathcal{L}_{\infty}$, and for $d=0$ the origin is globally finite-time stable and

$$
T\left(x_{0}\right) \leq-\frac{V_{0}^{-\mu}}{\alpha \mu},
$$

where $Q\left(V_{0}, x_{0}\right)=0$.

Remark 3 In order to satisfy the condition (11) the matrices $X$ and $\Lambda^{j}$ serve as solutions of the Lyapunov equation for the matrices $\tilde{A}_{0}$ and $A_{j}$ for $j=\overline{1, M}$, respectively. In this case it is important to choose appropriate representation of the system in the form (13) to better take the features of the LMIs (11). Indeed, without the use of the representation (13), the linear part of the system (5) in general case can be represented with nilpotent matrix $A_{0}$ (i.e. $\tilde{A}_{0}=A_{0}, \Psi^{j}=0$ ) for which the inequalities (11) are not feasible.

Similarly to [17], the matrices $\Xi^{k}$ for $k=\overline{0, M}$ are used in order to relax the stability requirements for each matrix $A_{k}$ for $k=\overline{0, M}$ by looking for their total influence on the system performance. The matrices $\Upsilon_{\eta, j}$ for $\eta=\overline{0, M-1}$ and $j=\overline{\eta+1, M}$ are introduced in order to reduce the conservatism of the condition

$$
\begin{aligned}
& Q_{1}+ {\left[\begin{array}{cc}
Q_{3} & \mathrm{O}_{(M+1) n \times n} \\
\mathrm{O}_{n \times(M+1) n} & \mathrm{O}_{n \times n}
\end{array}\right]+} \\
& \alpha\left[\begin{array}{cc}
Q_{2} & \mathrm{O}_{(M+1) n \times n} \\
\mathrm{O}_{n \times(M+1) n} & \mathrm{O}_{n \times n}
\end{array}\right] \leq 0 .
\end{aligned}
$$

Indeed, since for $\chi<\min \left\{\underline{s}_{i}^{2}, \bar{s}_{i}^{2}\right\} \lambda_{\min }(X)$ we have that $x_{i} V^{-r_{i}} \in\left[\underline{s}_{i}, \bar{s}_{i}\right] \backslash\{0\}$ for any $x_{i} \in \mathbb{R} \backslash\{0\}, i=\overline{1, n}$, then due to the sector properties (Assumption 1) we have that $\left(f_{i}^{j}\left(V^{-r_{i}} x_{i}\right)-\psi_{i}^{j} V^{-r_{i}} x_{i}\right) V^{-r_{i}} x_{i} \geq 0$ for $x \in \mathbb{R}^{n} \backslash$ $\{0\}$. In other words, all cross-terms, which appear in the off-diagonal blocks of the left-hand side of (14), should not be considered as additional "perturbations" in verification of the restriction (14) provided that they have a non-positive multiplier.

The advantages of using the representation (13) are based on the following observations:

- the linear term $\sum_{j=1}^{\rho} A_{j} \Psi^{j} x$ has slower dynamics in a neighbourhood of 0 with respect to $\sum_{j=1}^{\rho} A_{j} f^{j}(x)$;

- due to homogeneity property of the system and the chosen Lyapunov function, the global stability properties can be analysed in the vicinity of 0 , where the sector condition is satisfied for nonlinearities $f_{i}^{j}(x)-\psi_{i}^{j} x_{i}$, $i=\overline{1, n}, j=\overline{1, \rho}$.

Since for homogeneous system a local stability means the global one, the condition $\Xi_{0} \geq 0$ can be removed (locally at a vicinity of the origin, the quadratic term of the state, which is weighted by $\Xi_{0}$, is outperformed by the nonlinearities since $\mu<0)$ :

Corollary 2 Let all conditions of Corollary 1 be satisfied for $\alpha=0$ and a diagonal matrix $\Xi_{0} \in \mathbb{R}^{n \times n}$. Then the system (5) is ISS for $d \in \mathcal{L}_{\infty}$ and for $d=0$ the origin is globally finite-time stable.

Remark 4 Using the concept of local homogeneity the presented results can be applied to establish local finite-time stability for a more general class of systems.

Similarly to the representation (13), the same trick can be used for other terms of (5) with a slow dynamics in a neighbourhood of 0 , and the obtained results stay relevant. It is shown in the following corollary, that for stability analysis we can use the representation with the terms $A_{j}\left(f^{j}(x)-\right.$ $\left.\Psi^{j} x-\Phi^{j} h^{j}(x)\right)$, where $\Phi^{j} \in \mathbb{D}_{+}^{n}, j=\overline{1, \rho}$ and $h^{j}(x)$ have slow dynamics close to 0 .

Assume that in (5) the terms $A_{i} f^{i}(x(t)), i=\overline{\rho+1, \vartheta}$, $\vartheta \in[\rho+1, M]$ have slow dynamics close to 0 , and (11) is not feasible. For any $i=\overline{1, n}, j=\overline{1, \rho}, q=\overline{1, \vartheta-\rho}$ we 
have

$$
s f_{i}^{j}(s)>\phi_{i} f_{i}^{\rho+q}(s) s \quad \forall s \in\left[\underline{s}_{i}, \bar{s}_{i}\right] \backslash\{0\},
$$

where $\phi_{i} \in \mathbb{R}_{+}$and $-\infty<\underline{s}_{i}<0<\bar{s}_{i}<+\infty$ are as before. Define $\Phi=\operatorname{diag}\left\{\phi_{i}\right\}_{i=1}^{n} \in \mathbb{D}_{+}^{n}$ and $\hat{f}^{i}(x(t))=$ $\Phi f^{i}(x(t))$ for $i=\overline{\rho+1, \vartheta}$, then for $i=\overline{1, n}, j=\overline{1, \rho}$, $q=\overline{1, \vartheta-\rho}$ :

$$
s f_{i}^{j}(s)>\hat{f}_{i}^{\rho+q}(s) s \quad \forall s \in\left[\underline{s}_{i}, \bar{s}_{i}\right] \backslash\{0\}
$$

and by (9)

$s f_{i}^{j}(s)>\frac{1}{1+\vartheta-\rho}\left(\psi_{i}^{j} s+\sum_{q=1}^{\vartheta-\rho} \hat{f}_{i}^{\rho+q}(s)\right) s \forall s \in\left[\underline{s}_{i}, \bar{s}_{i}\right] \backslash\{0\}$.

Therefore, the following result can be used:

Corollary 3 Let Assumptions 1, 2 be satisfied. Let $\alpha \in$ $\mathbb{R}_{+}, \iota \in \mathbb{R}$ be chosen such that (11), (12) are satisfied for some $P=P^{T} \in \mathbb{R}^{n \times n} ; \Xi^{k}, \Lambda^{j}, \Upsilon_{\eta, l} \in \mathbb{D}_{+}^{n}, k=\overline{0, M}$, $j=\overline{1, M}, \eta=\overline{0, M-1}, l=\overline{\eta+1, M}$, with

$$
y=\left[\begin{array}{c}
D_{r}\left(V^{-1}\right) x \\
f^{1}\left(D_{r}\left(V^{-1}\right) x\right)-\frac{1}{1+\vartheta-\rho}\left[\Psi^{1} D_{r}\left(V^{-1}\right) x+\sum_{q=1}^{\vartheta-\rho} \hat{f}^{\rho+q}\left(D_{r}\left(V^{-1}\right) x\right)\right] \\
\vdots \\
f^{\rho}\left(D_{r}\left(V^{-1}\right) x\right)-\frac{1}{1+\vartheta-\rho}\left[\Psi^{\rho} D_{r}\left(V^{-1}\right) x+\sum_{q=1}^{\vartheta-\rho} \hat{f}^{\rho+q}\left(D_{r}\left(V^{-1}\right) x\right)\right] \\
\hat{f}^{\rho+1}\left(D_{r}\left(V^{-1}\right) x\right) \\
\vdots \\
\hat{f}^{\vartheta}\left(D_{r}\left(V^{-1}\right) x\right) \\
f^{\vartheta+1}\left(D_{r}\left(V^{-1}\right) x\right) \\
\vdots \\
f^{M}\left(D_{r}\left(V^{-1}\right) x\right)
\end{array}\right],
$$

$X=P+\frac{1}{1+\vartheta_{\tilde{\tau}} \rho} \sum_{j=1}^{\rho} \Lambda^{j} \Psi^{j}, \quad \tilde{A}_{0}=A_{0}+$ $\frac{1}{1+\vartheta-\rho} \sum_{j=1}^{\rho} A_{j} \Psi^{j}, \quad \tilde{A}_{i}=A_{i} \Phi^{-1}+\frac{1}{1+\underline{\varphi-\rho}} \sum_{j=1}^{\rho} A_{j}$ substituted instead of $A_{i}$ for $i=\overline{\rho+1, \vartheta}$, and $\tilde{\Lambda}^{j}=\Lambda^{j} \Phi^{-1}+\frac{1}{1+\vartheta-\rho} \sum_{q=1}^{\rho} \Lambda^{q}$ substituted instead of $\Lambda^{j}$ for $j=\overline{\rho+1, \vartheta}$.

Then the origin is globally finite-time stable and

$$
T\left(x_{0}\right) \leq-\frac{V_{0}^{-\mu}}{\alpha \mu}
$$

where $Q\left(V_{0}, x_{0}\right)=0$.

Remark 5 If in (5) there exists a locally bounded and piecewise continuous $f_{j}, j \in\{1, \ldots, M\}$, then under the assumption that the trajectories do not stay on the discontinuity set (except at the origin), the results above are preserved by interpreting the inequality for the derivative of Lyapunov function in the almost everywhere sense.

\section{NUMERICAL EXAMPLES}

Example 1 Consider the system

$$
\dot{x}=\left[\begin{array}{lll}
0 & 2 & 3 \\
0 & 0 & 0 \\
0 & 0 & 0
\end{array}\right] x+\left[\begin{array}{ccc}
-1 & 0 & 0 \\
0 & -2 & 1 \\
0 & 0.5 & -1
\end{array}\right]\left[\begin{array}{c}
\left|x_{1}\right|^{2 / 3} \operatorname{sign}\left(x_{1}\right) \\
\sqrt{\left|x_{2}\right|} \operatorname{sign}\left(x_{2}\right) \\
\frac{\sqrt{\left|x_{3}\right| \operatorname{sign}\left(x_{3}\right)}}{2+\operatorname{sign}\left(x_{3}\right)}
\end{array}\right]
$$

that is homogeneous of degree $\mu=-1$ with $r=\left[\begin{array}{ll}3 & 2\end{array}\right]$, i.e., Assuption 2 is satisfied. Due to $f^{1}(x)=\left[\begin{array}{c}\left|x_{1}\right|^{2 / 3} \operatorname{sign}\left(x_{1}\right) \\ \sqrt{\left|x_{2}\right|} \operatorname{sign}\left(x_{2}\right) \\ \frac{\sqrt{\left|x_{3}\right|} \operatorname{sign}\left(x_{3}\right)}{2+\operatorname{sign}\left(x_{3}\right)}\end{array}\right]$ is homogeneous of negative degree, Assumption 1 and (9) are satisfied with $j=1, i=\overline{1,3}$.

Let us represent the system in the form (13):

$$
\dot{x}=\left[\begin{array}{ccc}
-1 & 2 & 3 \\
0 & -1 & 1 \\
0 & 0.25 & -1
\end{array}\right] x+\left[\begin{array}{ccc}
-1 & 0 & 0 \\
0 & -2 & 1 \\
0 & 0.5 & -1
\end{array}\right]\left[\begin{array}{c}
\left|x_{1}\right|^{2 / 3} \operatorname{sign}\left(x_{1}\right)-x_{1} \\
\sqrt{\left|x_{2}\right| \operatorname{sign}\left(x_{2}\right)-0.5 x_{2}} \\
\frac{\sqrt{\left|x_{3}\right| \sin \left(x_{3}\right)}}{2+\operatorname{sign}\left(x_{3}\right)}-x_{3}
\end{array}\right]
$$

where for $\tilde{A}_{0}=\left[\begin{array}{ccc}-1 & 2 & 3 \\ 0 & -1 & 1 \\ 0 & 0.25 & -1\end{array}\right], A_{1}=\left[\begin{array}{ccc}-1 & 0 & 0 \\ 0 & -2 & 1 \\ 0 & 0.5 & -1\end{array}\right]$ and $\Psi^{1}=\left[\begin{array}{ccc}1 & 0 & 0 \\ 0 & 0.5 & 0 \\ 0 & 0 & 1\end{array}\right]$ the LMI (11) is feasible. Thus, the system is ISS and globally finite-time stable. The results of simulation with using the logarithmic scale are shown in Fig. 1 in order to demonstrate finite-time convergence rate of $\|x\|$.

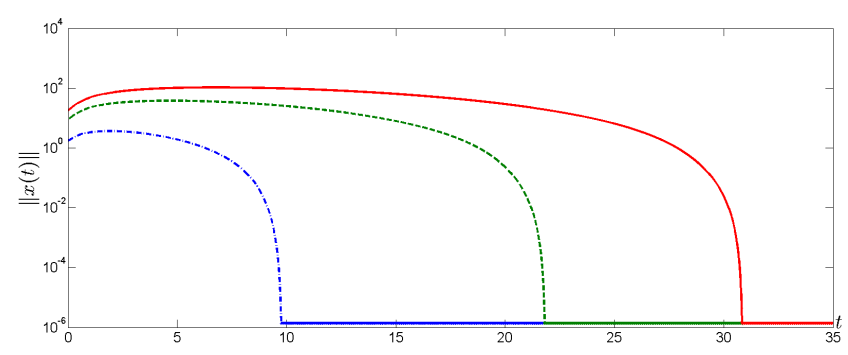

Fig. 1. The results of simulation for three different initial conditions

Example 2 Consider the system

$$
\dot{x}=\left[\begin{array}{ccc}
-1 & 0 & 0 \\
0 & -2 & 1 \\
0 & 0.5 & -1
\end{array}\right]\left[\begin{array}{c}
\left|x_{1}\right|^{2 / 3} \operatorname{sign}\left(x_{1}\right) \\
\sqrt{\left|x_{2}\right|} \operatorname{sign}\left(x_{2}\right) \\
\frac{\sqrt{\left|x_{3}\right| \operatorname{sign}\left(x_{3}\right)}}{2+\operatorname{sign}\left(x_{3}\right)}
\end{array}\right]+\left[\begin{array}{ccc}
0 & 1 & 0.2 \\
0 & 0 & 0 \\
0 & 0 & 0
\end{array}\right]\left[\begin{array}{c}
0 \\
x_{2}\left(\operatorname{sign}\left(x_{2}\right)+2\right) \\
x_{3}\left(\operatorname{sign}\left(x_{3}\right)+2\right)
\end{array}\right],
$$

where Assumptions 1, 2 are satisfied. Then, according to Corollary 3 let us represent the system as follows:

$$
\begin{aligned}
\dot{x}= & {\left[\begin{array}{ccc}
-1 & 0 & 0 \\
0 & -1 & 1 \\
0 & 0.25 & -1
\end{array}\right] x+} \\
& {\left[\begin{array}{ccc}
-1 & 0 & 0 \\
0 & -2 & 1 \\
0 & 0.5 & -1
\end{array}\right]\left[\begin{array}{c}
\left|x_{1}\right|^{2 / 3} \operatorname{sign}\left(x_{1}\right)-2 x_{1} \\
\sqrt{\left|x_{2}\right|} \operatorname{sign}\left(x_{2}\right)-0.5 x_{2}-0.5 x_{2}\left(\operatorname{sign}\left(x_{2}\right)+2\right) \\
\frac{\sqrt{\left|x_{3}\right| \sin \left(x_{3}\right)}-x_{3}-x_{3}\left(\operatorname{sign}\left(x_{3}\right)+2\right)}{2+\operatorname{sign}\left(x_{3}\right)} \\
x_{1}
\end{array}\right]+} \\
& {\left[\begin{array}{ccc}
-1 & 1 & 0.2 \\
0 & -1 & 1 \\
0 & 0.25 & -1
\end{array}\right]\left[\begin{array}{c}
x_{2}\left(\operatorname{sign}\left(x_{2}\right)+2\right) \\
x_{3}\left(\operatorname{sign}\left(x_{3}\right)+2\right)
\end{array}\right], }
\end{aligned}
$$

where the sector condition is satisfied in the vicinity of 0 . Then, for $\tilde{A}_{0}=\left[\begin{array}{ccc}-1 & 0 & 0 \\ 0 & -1 & 1 \\ 0 & 0.25 & -1\end{array}\right], A_{1}=\left[\begin{array}{ccc}-1 & 0 & 0 \\ 0 & -2 & 1 \\ 0 & 0.5 & -1\end{array}\right]$, $\tilde{A}_{2}=\left[\begin{array}{ccc}-1 & 1 & 0.2 \\ 0 & -1 & 1 \\ 0 & 0.25 & -1\end{array}\right]$ and $\Psi^{1}=\left[\begin{array}{ccc}1 & 0 & 0 \\ 0 & 0.5 & 0 \\ 0 & 0 & 1\end{array}\right]$ the LMI (11) is feasible, and the system is ISS and globally finite-time stable. 


\section{CONCLUSIONS}

In the paper, sufficient conditions of robust finite-time stability are presented for a class of homogeneous generalized Persidskii systems. The conditions are given in terms of LMI. The proposed approach extends the results of [17]: with the use of system representation (13), simple LMI-based stability conditions were extended on the class of homogeneous Persidskii systems; and nonasymptotic (finite-time) stability was studied. The proposed results allow to obtain settling time estimates. Possible directions for future research include an extension of the results for homogeneous Persidskii systems with positive degree and finite-time control and observer design.

\section{REFERENCES}

[1] V. Zubov, On systems of ordinary differential equations with generalized homogeneous right-hand sides (in Russian), Izvestia vuzov. Mathematica, vol. 1, pp. 80-88, 1958.

[2] E. Bernuau, A. Polyakov, D. Efimov, W. Perruquetti, Verification of ISS, iISS and IOSS properties applying weighted homogeneity, System and Control Letters, vol. 62, no. 12, pp. 1159-1167, 2013.

[3] A. Polyakov, Generalized Homogeneity in Systems and Control, Springer, 2020.

[4] A. Polyakov, D. Efimov, W. Perruquetti, Finite-time and fixed-time stabilization: Implicit Lyapunov function approach, Automatica, vol. 51, pp. 332-340, 2015.

[5] K. Zimenko, A. Polyakov, D. Efimov, On finite-time robust stabilization via nonlinear state feedback, International Journal of Robust and Nonlinear Control, vol. 28(16), pp. 4951-4965, 2018, DOI: $10.1002 /$ rnc. 4292.

[6] D. Efimov, A. Polyakov, W. Perruquetti and J.-P. Richard, Weighted homogeneity for time-delay systems: Finite-time and independent of delay stability, IEEE Trans. Automatic Control, vol. 61(1), pp. 210 215, 2016.

[7] K. Zimenko, D. Efimov, A. Polyakov, W. Perruquetti, A note on delay robustness for homogeneous systems with negative degree, Automatica, vol. 79, pp. 178-184, 2017, DOI: 10.1016/j.automatica.2017.01.036

[8] V. Andrieu, L. Praly, A. Astolfi, Homogeneous approximation, recursive observer and output feedback, SIAM Journal of Control and Optimization, vol. 47(4), pp. 1814-1850, 2008.

[9] A. Bacciotti, L. Rosier, Liapunov Functions and Stability in Control Theory, Springer, 2005.

[10] A. Polyakov, Sliding Mode Control Design Using Canonical Homogeneous Norm, Int. J. Robust. Nonlinear Control, pp. 1-20, DOI: 10.1002/rnc.4058, 2018

[11] M. Kawski, Geometric Homogeneity and Stabilization, in Proc. IFAC Nonlinear Control Symposium, A. Krener and D. Mayne, Eds., Lake Tahoe, CA, pp. 164-169, 1995.

[12] A. Levant, Homogeneity approach to high-order sliding mode design, Automatica, vol. 41, pp. 823-830, 2005, DOI: 10.1016/j.automatica.2004.11.029

[13] J.-B. Pomet, C. Samson, Time-varying exponential stabilization of nonholonomic systems in power form [dissertation], Villeneuved'Ascq, France: Inria, 1993.

[14] Y. Orlov, Finite Time Stability and Robust Control Synthesis of Uncertain Switched Systems, SIAM Journal of Control and Optimization, vol. 43(4), pp. 1253-1271, 2004.

[15] J. Hopfield, D. Tank, Computing with neural circuits: a model, Science, vol. 233, pp. 625-633, 1986.

[16] E. Sontag, Dealing with Complexity: a Neural Network Approach, London: Springer-Verlag, 1997, ch. Recurrent neural networks: Some systems-theoretic aspects, pp. 1-12.

[17] D. Efimov, A. Aleksandrov. On analysis of Persidskii systems and their implementations using LMIs. Automatica, In press.

[18] L. Ferreira, E. Kaszkurewicz, A. Bhaya, Solving systems of linear equations via gradient systems with discontinuous righthand sides: application to LS-SVM, IEEE Transactions on Neural Networks, vol. 16, no. 2, pp. 501-505, 2005.
[19] I. Karafyllis, Z.-P. Jiang, Stability and Stabilization of Nonlinear Systems, London, UK: Springer-Verlag London Ltd, 2011.

[20] A. Filippov, Differential equations with discontinuous right-hand sides, Dordrecht, Kluwer, 1988

[21] S. Bhat and D. Bernstein, Finite-time stability of continuous autonomous systems, SIAM Journal of Control and Optimization, vol 38(3), pp. 751-766, 2000.

[22] V.I. Korobov, A general approach to synthesis problem, Doklady Academii Nauk SSSR, vol. 248, pp. 1051-1063, 1979.

[23] J. Adamy, A. Flemming, Soft variable-structure controls: a survey, Automatica, vol. 40, pp. 1821-1844, 2004.

[24] W. Mei, D. Efimov, R. Ushirobira, Feedback synchronization in Persidskii systems, Proc. 21st IFAC World Congress, 2020.

[25] H. Silm, D. Efimov, W. Michiels, R. Ushirobira, J.-P. Richard, A simple finite-time distributed observer design for linear time-invariant systems, Systems and Control Letters, vol. 141, 2020. 